\title{
Post-euthanasia micro-computed tomography-based strain analysis is able to represent quasi-static in vivo behavior of whole vertebrae
}

Chetan Choudhari, Ryan Herblum, Margarete K Akens, Sara Moore, Michael Hardisty, and Cari M. Whyne

Version Post-Print/Accepted Manuscript

Citation Choudhari C, Herblum R, Akens MK, Moore S, Hardisty M, Whyne

(published version) CM. Post-euthanasia micro-computed tomography-based strain analysis is able to represent quasi-static in vivo behavior of whole vertebrae.Proc Inst Mech Eng H. 2016 Jul 15. doi:10.1177/0954411916658679. pii: 0954411916658679. [Epub ahead of print]. PMID: 27422827

Publisher's Statement The final published version of this article is available via SAGE

Publications via https://dx.doi.org/10.1177/0954411916658679.

Always cite the published version, so the author(s) will receive recognition through services that track citation counts, e.g. Scopus. If you need to cite the page number of the TSpace version (original manuscript or accepted manuscript) because you cannot access the published version, then cite the TSpace version in addition to the published version using the permanent URI (handle) found on the record page. 


\title{
Post-euthanasia micro-CT based strain analysis is able to represent quasi-static in vivo behavior of whole vertebrae
}

\author{
Chetan Choudhari ${ }^{1,4}$, Ryan Herblum ${ }^{1,4}$, Margarete K. Akens ${ }^{2,3}$, Sara Moore ${ }^{1}$, Michael R. \\ Hardisty $^{1}$, Cari M. Whyne ${ }^{1,3,4}$. \\ ${ }^{1}$ Sunnybrook Research Institute, Toronto, ON, Canada, ${ }^{2}$ TECHNA Institute, University Health \\ Network, Toronto, ON, Canada, ${ }^{3}$ Department of Surgery, Toronto, ON, Canada, ${ }^{4} I B B M E$, \\ Toronto, ON, Canada.
}

Corresponding Author: Dr. Cari Whyne

Sunnybrook Health Sciences Centre

2075 Bayview Ave., Room S620

Toronto, ON

M4N 3M5

Phone: 416-480-6100, ext. 5056

Email: cari.whyne@sunnybrook.ca

\begin{abstract}
Three-dimensional image-based strain measurement in whole bones allows representation of physiological, albeit quasistatic, loading conditions. However, such work to date has been limited to specimens postmortem. The main purpose of this study is to verify the efficacy of deformable image registration of post-euthanasia strain to characterize the in vivo mechanical behavior of rat vertebrae. A micro-computed tomography-compatible custom loading device was used to apply $75 \mathrm{~N}$ load to a three-level caudal motion segment of a healthy rat. Loaded and unloaded micro-computed tomography scans were acquired in vivo and post-sacrifice. A microcomputed tomography-based deformable image registration algorithm was used to calculate vertebral strains live and post-euthanasia. No significant difference was found in the in vivo strains (20.01160.001) and ex vivo strains (20.01260.001) obtained from the comparisons of loaded and unloaded images $(\mathrm{p}=0.3)$. Comparisons between unloaded-unloaded and loadedloaded scans yielded significantly lower axial strains, representing the error of the method. Qualitatively, high strains were observed adjacent to growth plate regions in evaluating the
\end{abstract}


loaded-unloaded images. Strain patterns in the loaded-loaded and unloaded-unloaded scans were inconsistent as would be expected in representing noise. Overall, live and dead loaded to unloaded comparisons yielded similar strain patterns and magnitudes. Point-wise differences in axial strain fields also supported this observation. This study demonstrated a proof of concept, suggesting that post-euthanasia micro-computed tomography-based strain analysis is able to represent the in vivo quasi-static behavior of rat tail vertebrae.

Keywords: Biomechanical Testing/ Analysis, Bone Biomechanics, Bone Properties, Computed Tomography [Ct] Analysis, Imaging [Biomechanics], Spine Biomechanics, Strain Analysis/ Testing [Biomechanics] 


\section{Introduction}

Deformable image registration (DIR) tracks the deformation of microstructural features and patterns within images of a sample acquired under unloaded and loaded configurations to yield full field strain measurements (1). In evaluating bone strain, DIR has been utilized to study cortical and trabecular specimens $(2,3)$, with some application to whole bones (4-8) allowing the representation of more physiological, albeit quasi-static ex vivo, loading conditions. The aim of this study was to demonstrate the ability of ex vivo (post-euthanasia) modeling to represent the in vivo quasi static behavior of a whole vertebra through image-based strain analysis. It is hypothesized that post-euthanasia $\mu \mathrm{CT}$ image-based strain analysis represents the in vivo quasistatic behavior of whole bones.

\section{Methods}

\section{DIR algorithm}

An intensity matching DIR algorithm was previously developed and validated in which unloaded and loaded $\mu \mathrm{CT}$ scans are registered using affine mappings of sub-regions (4). The initial registration proceeds iteratively, optimizing the affine mapping based upon a normalized mutual information metric. The unloaded scan is then split along the three axes, yielding 8 pieces. Each piece is individually registered to the loaded scan, using the affine transform from the previous registration as the initial guess. The division and registration of sub-pieces continues until a final level of registration is reached. The affine transform is then used to calculate the displacement of the center of each registered sub-region. Continuum level strain fields (strain resolution $892.5 \mu \mathrm{mx} 772.5 \mu \mathrm{mx} 1157.5 \mu \mathrm{m})$ were calculated using these displacements; regions falling outside of the vertebral body and those regions that did not register were excluded (Figure 1).

\section{Loading}

A $\mu \mathrm{CT}$ compatible custom loading device was used to apply axial compressive load of $75 \mathrm{~N}$ to a 3-level vertebral motion segment in the rat tail. This load was previously demonstrated to induce detectable strain in the vertebrae without fracture (7). A radiolucent jig (Figure 2) was attached to the $5^{\text {th }}$ to $7^{\text {th }}$ caudal vertebrae in the anaesthetized rat via percutaneous pins. Quasi-static load 
was applied to the rat tail manually through the threaded tube. The vertebrae were preconditioned with application and release of smaller loads, before applying the desired load $(75 N)$. After each load application or removal, the load on the tail was allowed to stabilize prior to scanning.

\section{Imaging}

In vivo loaded $\mu \mathrm{CT}$ imaging $(80 \mathrm{kV}, 13.3 \mu \mathrm{m}$ voxel size, Inveon $\mathrm{CT}$, Siemens Healthcare) was performed on one healthy female Sprague Dawley rat (17 weeks old, 300g) under general anesthesia with an average scan time of 90 minutes. After euthanasia, dead loaded and unloaded $\mu \mathrm{CT}$ images were acquired. No issues originating from the radiation dose were observed, thermal or otherwise during experimentation. A total of $7 \mu \mathrm{CT}$ scans were acquired in the given sequence and configurations: live unloaded[0], live under load [1], dead under load [2, 3], dead unloaded $[4,5]$ and dead under load [6]. The live unloaded imaging data [0] was acquired however due to a motion artifact, not noticed till after reconstruction the volume was not unusable for analysis.

\section{Analysis}

The middle vertebrae (6th caudal) from all scans were cropped and the bone regions segmented using a common global threshold to allow for better registration. Each loaded scan was registered with each unloaded scan to determine the load induced strain. Registration of scans under similar load configurations (loaded-loaded and unloaded-unloaded) were also performed to determine the zero strain error. Axial strain distributions in the rat-tail vertebrae were characterized by calculating the mean strain, median strain and the $10^{\text {th }}$ and $90^{\text {th }}$ percentile strains. T-tests were used to evaluate differences between strain values in considering live-todead and dead-to-dead images. A Bonferroni correction was used to adjust the significance level $(\alpha=0.016)$. Qualitative and quantitative comparisons of the strain patterns generated within the vertebrae were also performed by visual inspection of results and calculation of direct pointwise differences between strain fields. 


\section{Results}

Mean axial compressive strains $(-0.012 \pm 0.001$ (Table 1)) were induced in the vertebral bodies as measured in the loaded-unloaded scan configurations [1-4, 1-5, 2-4, 2-5, 6-4 and 6-5]. Comparisons between unloaded-unloaded [4-5] and loaded-loaded [1-2, 1-3, 1-6, 2-3, 2-6 and 36] scans yielded mean axial strains near zero strain as expected, representative of the error in the method (Table 2, Figure 3a). Load induced strain fields were not significantly different between live configurations [1-4, 1-5] or dead configurations[2-4, 2-5, 3-4, 3-5, 6-4 and 6-5] with respect to the mean strains generated (Figure $3 b$ ) and when evaluating point-wise differences in axial strain fields (Table 1). Differences between live and dead strain patterns were not larger than those differences arising from rescanning or reloading the same dead specimen. In comparing images acquired under equivalent loading conditions, (loaded-loaded and unloaded-unloaded) similar strain errors were found in assessing dead to dead [2-3, 3-6 and 2-6] images vs. dead to live [1-2, 1-3 and 1-6] images (Figure 3c). However, loaded-loaded comparisons yielded higher mean strains than the unloaded-unloaded comparison. The median and $90 \%$ strain values were observed to follow the same pattern as the mean strains. The average $10 \%$ strains for all comparisons were not significantly different.

Qualitatively, the strain patterns were similar in comparing live and dead loaded-unloaded images, with high strains adjacent to the inferior and superior growth plates (Figure 4). Strain patterns in the loaded-loaded and unloaded-unloaded scans were inconsistent as would be expected since it is strain error represented in these images (Figure 5).

\section{Discussion}

In vivo application of load to a single rat tail vertebra was successfully accomplished within a $\mu \mathrm{CT}$ scanner allowing the comparison of live and post-euthanasia strains. No significant differences were observed in the mean live/dead strains obtained through loaded-unloaded comparisons. The average strains were in the range of previously reported ex vivo strains in rat tail vertebrae loaded in a similar configuration (5). The difference between the live/dead results was within the variance found within each group. 
The strain obtained from the registration of loaded-loaded images [-0.003] was found to be 50 times higher as compared to that of unloaded-unloaded images [-0.00006]. Hardisty et al. observed increased error in strain calculation with increased load (4). Slight changes in the load distribution may have also occurred during load reapplication for the final scans. Small amounts of stress relaxation in the tails in response to the applied load may also have contributed to these variations. Nonetheless, the strains obtained from comparisons of images under similar loading conditions are in the range of standard deviations of the strains obtained from loaded-unloaded comparisons.

Measured error in strain calculation, represented by comparison of unloaded-unloaded images, was found to be -0.00006 (accuracy), with a standard deviation of 0.004 (precision). Previous work utilizing this DIR routine was able to measure strain in whole ex vivo rat vertebrae with accuracy and precision of 0.0003 and 0.0004 respectively, based on an unloaded zero strain case (4). Point wise differences in load induced strain fields were on average 0.0048 (Table 1). This point wise difference includes errors in zero strain ( 0.00006) plus registration error $(10-25 \%$ of strain magnitude (4) and changes in intensity distributions from repeat scanning (which seem to accumulate to $\sim 32 \%$ of the strain magnitude). The accuracy and precision of strain calculations are influenced by the size of the region registered, where an increase in the size of the registered region leads to increased precision in the strain field, but decreased accuracy (5). The apparent strains determined through DIR ( 0.012) are similar to those found previously in trabecular bone (9).

DIR has several advantages over some of the traditional methods for resolving bone strain. Biomechanical testing of whole bone samples is often used but only resolves bulk strain $(10,11)$. Strain gage application on small bone structures is challenging and is limited to surface strain measurements (12). Finite element analysis (FEA) can determine full field strain patterns in whole bones, however, accurate knowledge of geometry, material properties and boundary conditions is essential for a successful FEA (13). Image registration requires no assumptions and can be directly applied to two sets of images to determine full field strain (4) or be used in combination with FEA to set complex displacement boundary conditions (14).

A limitation of this study was lack of comparison with an in vivo unloaded scan. The initial experimental design included this acquisition; however, the in vivo unloaded $\mu \mathrm{CT}$ image was 
rendered unusable due to a motion artifact. Also, while the repeated measures analysis required considerable computational effort, this study was focused on a single sample. This work demonstrates a proof of concept and subsequent work may evaluate variability among multiple specimens and loading conditions.

The DIR method relies on contrast between bone and marrow to accurately register images (4). Yet it is the structure size required to accurately calculate the displacement field that in fact limits the resolution (15). The limited resolution causes the trabeculae adjacent to the more compliant growth plates to appear highly strained. The limited spatial resolution of DIR results in measuring only the average strain within individually registered regions based on the pattern within the region. This smooths the strain field and yields a strain resolution that is lower than the voxel size of the image, resulting in a limited ability of the technique to accurately locate small regions of high strains at an elevated risk of damage. Efforts are currently being made in our group to improve the spatial resolution of the algorithm using feature based registration.

Overall, the intensity based DIR module demonstrated that live and dead loaded to unloaded comparisons yielded similar strains, concentrated along the inferior and superior growth plates. This technical proof of concept study suggests that post-euthanasia $\mu \mathrm{CT}$ based strain analysis is able to represent the in vivo quasi-static behaviour of rat-tail vertebrae.

\section{Acknowledgements}

This research was supported by Canadian Institute of Health Research (\#MOP68911). 


\section{FIGURES}

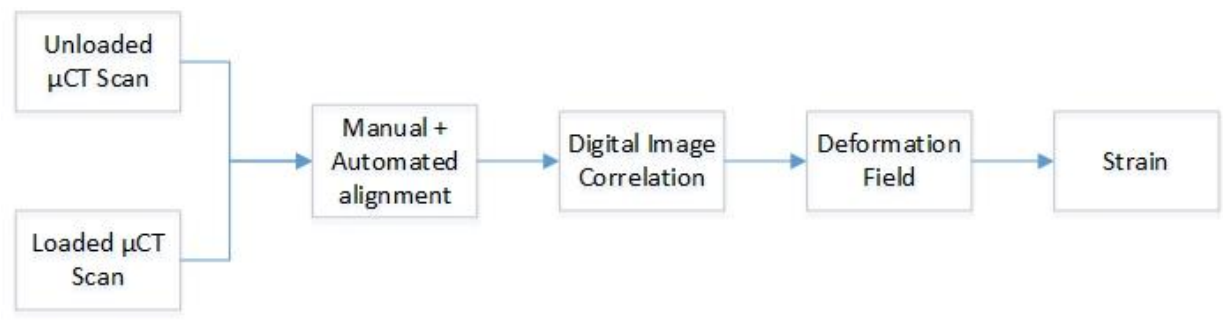

Figure 1: Deformable image registration algorithm determines strain by aligning and comparing scans of the same sample with and without load (Hojjat 2011).

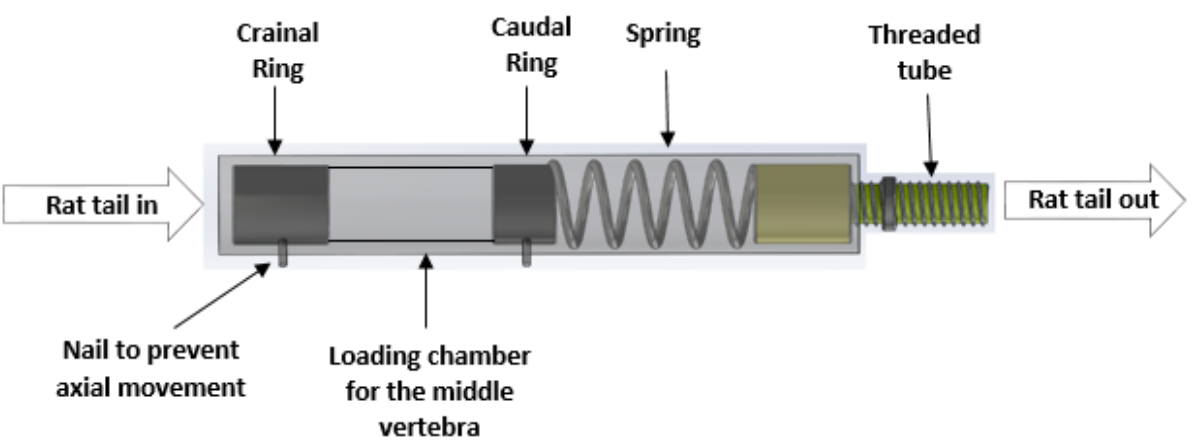

Figure 2: Micro-CT compatible loading jig used to apply an axial compressive load to the 6th caudal vertebra in the rat tail. Nails were inserted through the adjacent vertebrae and into the cranial and caudal rings. The rings can slide against the outer clear tube. This assembly ensures axial motion and prevents lateral movement of the vertebrae during loading. The cranial ring is fixed with a nail as shown to the outer tube, while the caudal ring is free to translate during load application. 


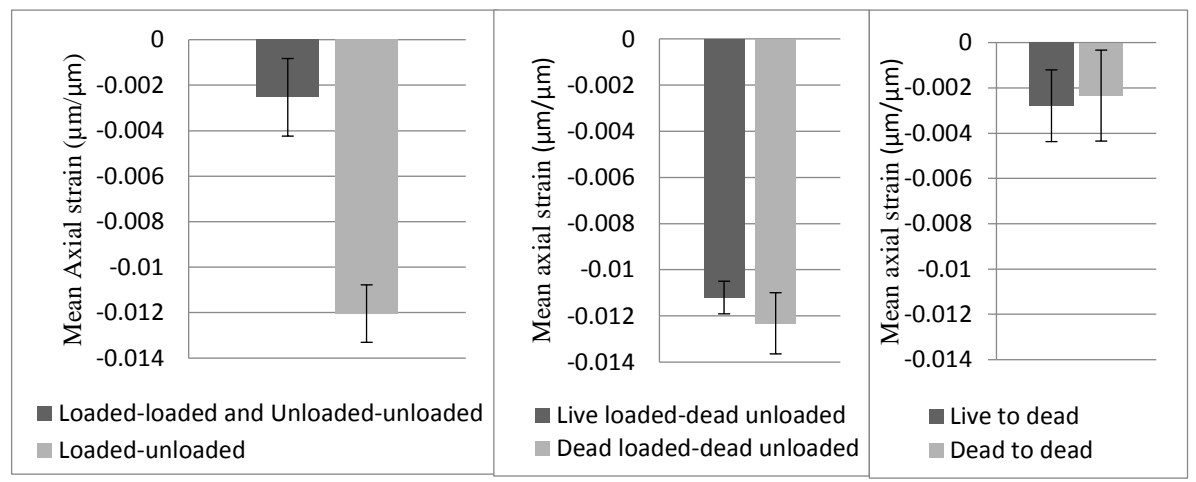

a)

b)

c)

Figure 3: Mean axial strains $(\mu \mathrm{m} / \mu \mathrm{m})$ obtained from deformable registration (* represents p-value<0.016). a) Strains obtained from all loaded-unloaded and all loadedloaded/unloaded-unloaded comparisons. b) Comparison of live and dead strains. c) Live and dead strains for scans under equivalent loading conditions 


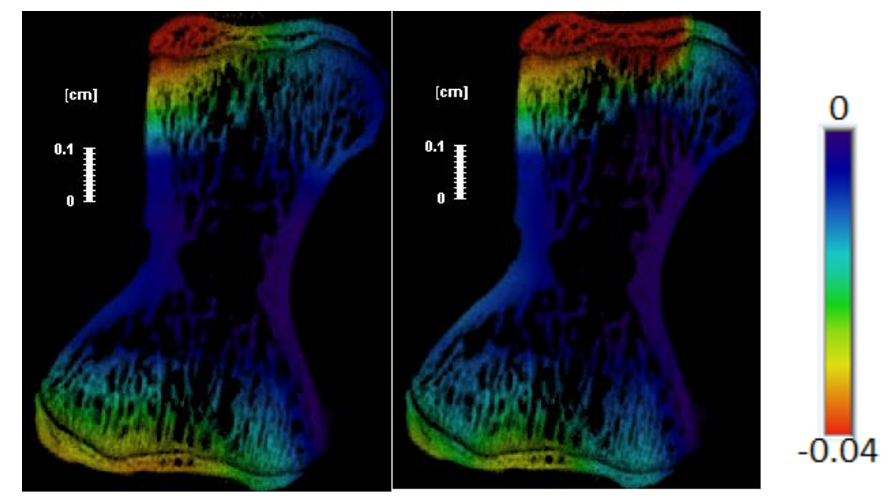

Figure 4: Axial strain patterns in loaded-unloaded strain registrations. The strain is concentrated mostly around the endplate regions. a) Dead-loaded to dead-unloaded comparison b) Live-loaded to dead-unloaded comparison

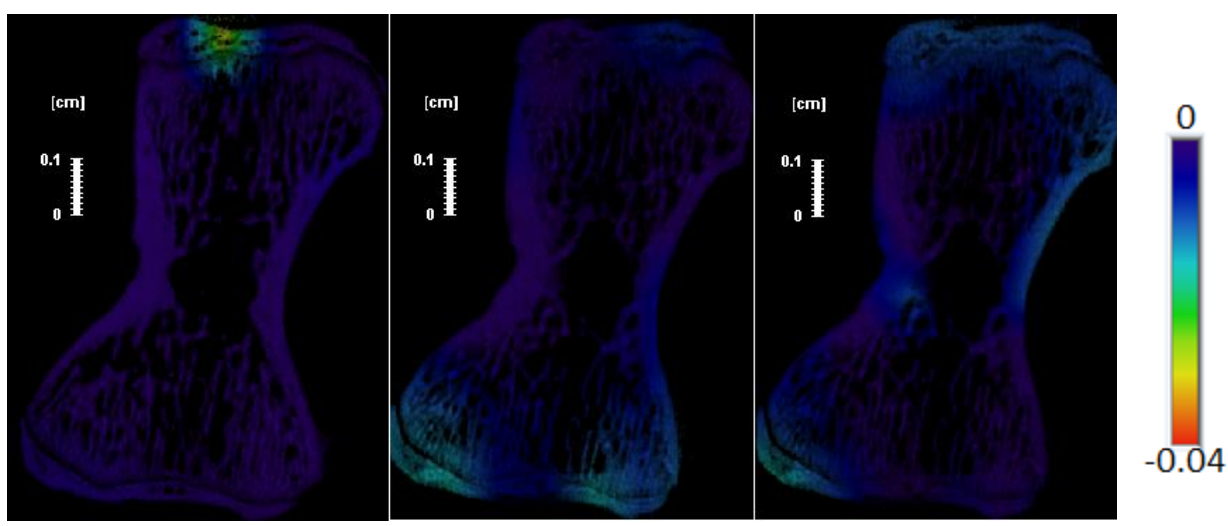

Figure 5: Axial strain patterns generated by comparison of scans under equivalent loading conditions. a) Live-loaded to dead loaded b) Dead-loaded to dead-loaded c) Dead-unloaded to dead-unloaded 
TABLES

Table I. Strairs $(\mu \mathrm{m} / \mu \mathrm{m})$ obtained from the comparisons of loaded-unloaded inages.

\begin{tabular}{llllll}
\hline & Mean strain & $10 \%$ strain & Median strain & $90 \%$ strain & $\begin{array}{l}\text { Mean absolute } \\
\text { difference }\end{array}$ \\
\hline Live-loaded to dend urloaded & $-0.011 \pm 0.001$ & $0.004 \pm 0.002$ & $-0.013 \pm 0.002$ & $-0.023 \pm 0.001$ & $0.0058 \pm 0.0013$ \\
Dead-loaded to dead-urloaded & $-0.012 \pm 0.001$ & $0.004 \pm 0.001$ & $-0.013 \pm 0.002$ & $-0.027 \pm 0.004$ & $0.0038 \pm 0.0012$ \\
\hline
\end{tabular}

Table 2. Strairs $(\mu \mathrm{m} / \mu \mathrm{m})$ obtained from the comparisons of images under similar loading conditions.

\begin{tabular}{lllll}
\hline & Mean strain & $10 \%$ strain & Median strain & $90 \%$ strain \\
\hline Live-loaded to dead loaded & $-0.003 \pm 0.002$ & $0.005 \pm 0.002$ & $-0.003 \pm 0.002$ & $-0.010 \pm 0.004$ \\
Dead-loaded to dead-loaded & $-0.003 \pm 0.002$ & $0.007 \pm 0.003$ & $-0.003 \pm 0.002$ & $-0.012 \pm 0.003$ \\
Dead-urloaded to dead-urloaded & -0.00006 & 0.004 & -0.0001 & -0.004 \\
\hline
\end{tabular}

